\title{
THE INCIDENCE OF DWARF NOVAE IN LARGE AREA TRANSIENT SEARCHES
}

\author{
A. Rau, ${ }^{1}$ R. Schwarz, ${ }^{2}$ S. R. Kulkarni, ${ }^{1}$ E. O. Ofek, ${ }^{1}$ M. M. Kasliwal, ${ }^{1}$ C. Brinkworth, ${ }^{3}$ \\ S. B. Cenko, ${ }^{4}$ Y. Lipkin, ${ }^{5}$ And A. M. Soderberg ${ }^{1}$ \\ Received 2006 October 12; accepted 2007 April 9
}

\begin{abstract}
Understanding and quantifying the contribution of known classes of transient and variable sources is an important lesson to be learned from the manifold of precursor programs of the near-future large synoptic sky survey programs such as SkyMapper, Pan-STARRS, and LSST. With this goal in mind, we undertook photometric and spectroscopic follow-up observations of four recently reported unidentified transients. For two sources, WFI J132813.7-214237 and WFI J161953.3+031909, we show that unfortunate coincidences led to their previous designation as transients. While the former is now interpreted as the spatial coincidence of a solar system object with a faint background star, the latter is merely a cataclysmic variable unfortunately caught in and out of eclipse. The third candidate, ROTSE3 J160213.1-021311.7, is identified as an SU UMa type dwarf nova with quiescent brightness of $R \sim 22.7$ and an outburst amplitude of $\sim 5$ mag. The fourth event, SDSS-SN 15207, similarly shows evidence for a dwarf nova origin. Our main conclusion is that cataclysmic variables in their various avatars will contribute moderately to the population of transient objects.
\end{abstract}

Subject headings: binaries: eclipsing — novae, cataclysmic variables — stars: dwarf novae

Online material: color figures

\section{INTRODUCTION}

The study of transient and variable phenomena has historically been one of the main drivers of optical astronomy. This field is now widely expected to undergo a revival, thanks to the dawn of dedicated facilities, such as SkyMapper (Schmidt et al. 2005), the Panoramic Survey Telescope and Rapid Response System (PanSTARRS; Kaiser et al. 2002), and the Large Synoptic Survey Telescope (LSST; Tyson 2005). A number of precursor surveys (e.g., Becker et al. 2004; Rykoff et al. 2005; Sako et al. 2005; Rau et al. 2006; Astier et al. 2006; Malacrino et al. 2006) are already informing us of the difficulty of finding genuine new classes of transients. Solar system objects (Malacrino \& Atteia 2006) and flares from M dwarfs (Becker et al. 2004; Kulkarni \& Rau 2006) are now demonstrated to be the most common variable objects.

In this paper we report on follow-up observations of four optical transients discovered in the ROTSE-III sky patrol (Rykoff et al. 2006), the MPG/ESO Wide Field Imager (WFI) search for orphan afterglows (Rau et al. 2006), and the SDSS supernovae search. Our findings show that cataclysmic variables (CVs) in general form an additional thin layer of foreground, which also must be pierced through before we can discover new classes of optical transients.

\section{ROTSE3 J160213.1-021311.7}

\subsection{Discovery}

The transient was discovered in ROTSE-III sky patrol fields on 2006 May 28.94 UT at an unfiltered magnitude of $18.2 \pm 0.2$

\footnotetext{
1 Caltech Optical Observatories, California Institute of Technology, MS 105-24, Pasadena, CA 91125; arne@astro.caltech.edu.

2 Astrophysical Institute Potsdam, An der Sternwarte 16, 14482 Potsdam, Germany.

3 Spitzer Science Center, California Institute of Technology, Pasadena, CA 91125 .

4 Space Radiation Laboratory, California Institute of Technology, Pasadena, CA 91125.

5 School of Physics and Astronomy and Wise Observatory, Tel-Aviv University, Tel-Aviv 69978, Israel.
}

(Rykoff et al. 2006). No counterpart was found in previous ROTSE-III observations, the Digitized Sky Survey, nor the Two Micron All Sky Survey. Following the discovery, the source brightened by $\sim 1.4$ mag within a day and quickly faded back to around 18th magnitude, where it seemed to stabilize for several days. Observations with the $1.5 \mathrm{~m}$ Russian-Turkish Telescope in Bakyrlytepe, Turkey, on 2006 June 1.88 UT detected the source at $B=18.96 \pm 0.02, V=18.31 \pm 0.02$, and $R_{\mathrm{C}}=17.69 \pm$ 0.02 (Rykoff et al. 2006). The $V-R$ color of 0.6 mag was commented to be redder than expected for an outburst of a cataclysmic variable. Thus, the source identification remained uncertain.

\subsection{Photometry}

Our photometric follow-up of the transient (Fig. 1) started on 2006 June 4.95 UT, 2 days after the discovery was announced and 6 days after the discovery itself. Our first epoch of observations were obtained with the $1 \mathrm{~m}$ telescope at the Wise Observatory, Israel, in the $B, V, R$, and $I$ bands. Monitoring in $g$ and $R$ with the automatic Palomar 60 inch telescope (P60) began 2 days later, and a final epoch was obtained with the Large Field Camera at the Palomar 200 inch telescope (P200). A summary of the observations is presented in Table 1 .

The first imaging epoch found the source at a brightness of $B=19.83 \pm 0.12, V=19.15 \pm 0.08, R=18.70 \pm 0.05$, and $I=18.81 \pm 0.08$. The subsequent observations with $\mathrm{P} 60$ traced the decay, which was interrupted by several rebrightening phases with amplitudes of $\Delta M=0.2-0.6 \mathrm{mag}$ and color changes from $g-R \sim 0.8$ to $\sim 1.2$ mag during the variations (Fig. 2 ). The total Galactic foreground extinction in the direction of the candidate is $E(B-V)=0.276$ (Schlegel et al. 1998).

During the first nights of P60 observations the brightness of the transient allowed a variability study on short timescales using the individual consecutive $150 \mathrm{~s} R$-band exposures (Fig. 2, inset).

We inspected images generated by the Near-Earth Asteroid Tracking (NEAT) program ${ }^{6}$ for past outburst episodes at the

${ }^{6}$ See http://skys.gsfc.nasa.gov/skymorph/obs.html. 


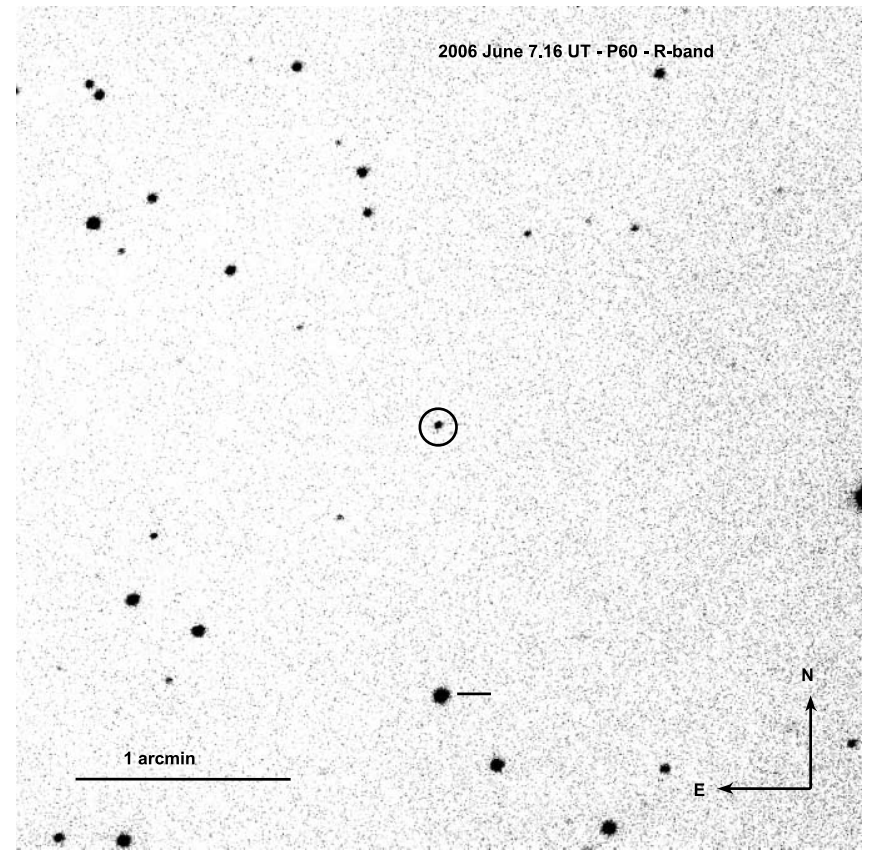

FIG. 1.-P60 image of the field around ROTSE3 J160213.1-021311.7 taken on 2006 June 7 . Astrometry was performed based on USNO-B1.0 positions of 10 stars in the neighborhood of the transient. The coordinates of the transient (circle) are $\alpha_{\mathrm{J} 2000.0}=16^{\mathrm{h}} 02^{\mathrm{m}} 13.06^{\mathrm{s}}, \delta_{\mathrm{J} 2000}=-02^{\circ} 13^{\prime} 12.4^{\prime \prime}$. A comparison star with $R=16$ mag located $0.9^{\prime \prime}$ west and $75.6^{\prime \prime}$ south at $\alpha_{\mathrm{J} 2000.0}=16^{\mathrm{h}} 02^{\mathrm{m}} 13.00^{\mathrm{s}}$, $\delta_{\mathrm{J} 2000.0}=-02^{\circ} 14^{\prime} 28.0^{\prime \prime}$ is marked by the horizontal dash.

TABLE 1

ROTSE3 J160213.1-021311.7 IMAGING LoG

\begin{tabular}{|c|c|c|c|c|}
\hline MJD & Telescope & Filter & $\begin{array}{c}\text { Exposure } \\
\text { (s) }\end{array}$ & $\begin{array}{l}\text { Brightness } \\
\quad \text { (mag) }\end{array}$ \\
\hline 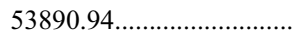 & Wise & $B$ & 840 & $19.83 \pm 0.12$ \\
\hline 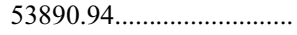 & Wise & V & 600 & $19.15 \pm 0.08$ \\
\hline 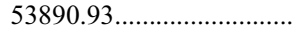 & Wise & $R$ & 600 & $18.70 \pm 0.05$ \\
\hline $53890.96 \ldots \ldots$. & Wise & $I$ & 600 & $18.81 \pm 0.08$ \\
\hline $53893.19 \ldots \ldots \ldots \ldots \ldots \ldots \ldots \ldots$ & P60 & $g$ & 1350 & $19.16 \pm 0.08$ \\
\hline $53893.19 \ldots \ldots \ldots \ldots \ldots \ldots \ldots$ & $\mathrm{P} 60$ & $R$ & 1350 & $18.42 \pm 0.05$ \\
\hline 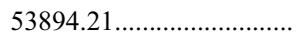 & P60 & $g$ & 1350 & $20.01 \pm 0.09$ \\
\hline $53894.21 \ldots \ldots$ & P60 & $R$ & 1350 & $18.79 \pm 0.05$ \\
\hline 53895.22. & $\mathrm{P} 60$ & $g$ & 1350 & $20.01 \pm 0.11$ \\
\hline 53895.22. & $\mathrm{P} 60$ & $R$ & 1350 & $19.22 \pm 0.07$ \\
\hline $53896.26 \ldots \ldots$ & P60 & $g$ & 1350 & $19.86 \pm 0.15$ \\
\hline $53896.26 \ldots \ldots \ldots \ldots \ldots$ & P60 & $R$ & 1350 & $19.05 \pm 0.09$ \\
\hline $53898.21 \ldots \ldots \ldots \ldots \ldots \ldots \ldots$ & P60 & $g$ & 1350 & $20.82 \pm 0.29$ \\
\hline 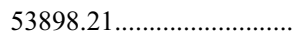 & $\mathrm{P} 60$ & $R$ & 1350 & $20.34 \pm 0.16$ \\
\hline $53899.25 \ldots \ldots \ldots \ldots \ldots \ldots \ldots \ldots \ldots \ldots \ldots \ldots$ & $\mathrm{P} 60$ & $g$ & 1350 & $20.84 \pm 0.22$ \\
\hline $53899.25 \ldots \ldots \ldots \ldots \ldots \ldots$ & $\mathrm{P} 60$ & $R$ & 1350 & $19.78 \pm 0.11$ \\
\hline 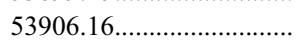 & $\mathrm{P} 60$ & $g$ & 1350 & $22.26 \pm 0.21$ \\
\hline $53906.16 \ldots \ldots \ldots \ldots \ldots \ldots \ldots$ & P 60 & $R$ & 1350 & $21.29 \pm 0.22$ \\
\hline $53908.20 \ldots \ldots \ldots \ldots \ldots \ldots \ldots \ldots \ldots \ldots$ & $\mathrm{P} 60$ & $R$ & 1350 & $21.43 \pm 0.19$ \\
\hline $53914.29 \ldots \ldots \ldots \ldots \ldots \ldots \ldots \ldots \ldots \ldots \ldots \ldots \ldots$ & $\mathrm{P} 60$ & $g$ & 1350 & $23.10 \pm 0.40$ \\
\hline $53914.29 \ldots \ldots \ldots \ldots \ldots \ldots$ & $\mathrm{P} 60$ & $R$ & 1200 & $21.87 \pm 0.29$ \\
\hline $53929.19 \ldots \ldots \ldots \ldots \ldots$ & $\mathrm{P} 60$ & $R$ & 2700 & $22.53 \pm 0.37$ \\
\hline 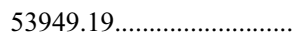 & $\mathrm{P} 60$ & $R$ & 2700 & $22.18 \pm 0.28$ \\
\hline $53968.23 \ldots \ldots \ldots \ldots \ldots$ & P200 & $g^{\prime}$ & 600 & $23.5 \pm 0.2$ \\
\hline $53968.24 \ldots \ldots \ldots \ldots \ldots \ldots \ldots \ldots$ & P200 & $r^{\prime}$ & 600 & $22.7 \pm 0.1$ \\
\hline
\end{tabular}

Note.-All magnitudes are given in the Vega system.

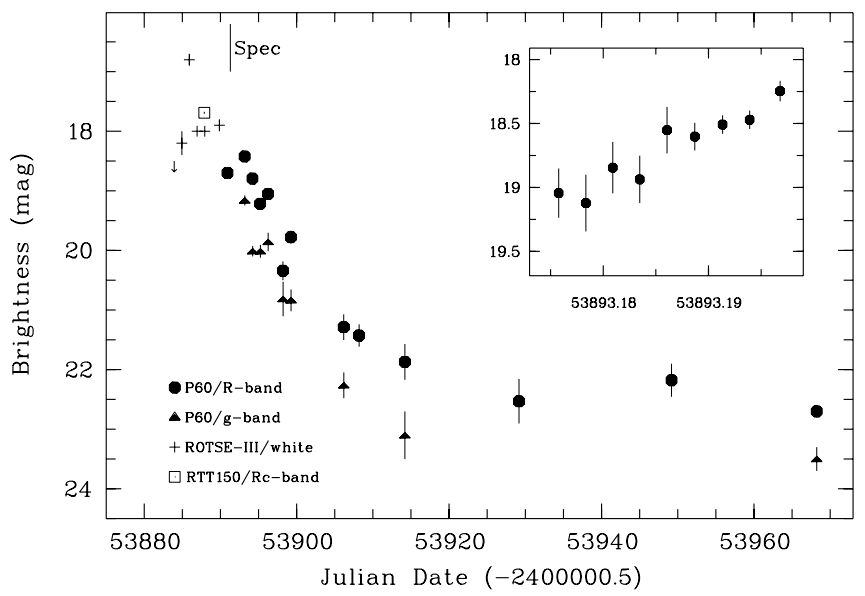

FIG. 2. - P60 light curve of ROTSE3 J160213.1-021311.7. Photometric calibrations ( Vega system) were obtained using observations of the Landolt standard star SA 108-475 (Landolt 1992). A clear monotonic brightening by $\sim 0.8 \mathrm{mag}$ over about 30 minutes was observed on June 7 (inset). Variability was also detected on June 8-10 at lower significance. The epoch of our DBSP spectroscopy is indicated by the vertical dash.

position of the transient. No source was detected in 17 epochs between 1998 June and 2003 July with limiting magnitudes (roughly $R$ band) of 20-21.5. Similarly, no X-ray counterpart was found in the ROSAT All-Sky Survey ${ }^{7}$ with a limit of $6 \times$ $10^{-3}$ counts s$^{-1}(0.1-2.4 \mathrm{keV})$. We conclude that the source is mostly in a quiescent state and rarely undergoes outbursts.

\subsection{Spectroscopy}

Spectroscopy was obtained with the Double-Beam Spectrograph (DBSP) mounted on the Hale $5 \mathrm{~m}$ telescope at Palomar Observatory (see Table 2). DBSP, as suggested by the name, operates with a dichroic, and spectra can be obtained in two (blue and red) channels simultaneously. The spectrum (Fig. 3) shows a flat continuum with strong emission features which we identify

\footnotetext{
${ }^{7}$ See http://www.xray.mpe.mpg.de/cgi-bin/rosat/rosat-survey.
}

TABLE 2

Log of the Spectroscopic Observations

\begin{tabular}{clcc}
\hline \hline $\begin{array}{c}\text { Date } \\
(1)\end{array}$ & \multicolumn{1}{c}{$\begin{array}{c}\text { Exposure } \\
(2)\end{array}$} & $\begin{array}{c}\text { Setup } \\
(3)\end{array}$ & $\begin{array}{c}(\mathrm{s}) \\
(4)\end{array}$ \\
\hline & \multicolumn{2}{c}{ ROTSE3 J160213.1-021311.7 } \\
\hline 2006 Jun 5 ............. & Hale 5 m DBSP & $300 / 3990$ & 2100 \\
& Hale 5 m DBSP & $316 / 7500$ & 2100
\end{tabular}

\begin{tabular}{llcl}
\hline \multicolumn{4}{c}{ WFI J161953.3+031909 } \\
\hline 2006 Apr 22........... & Keck LRIS & $400 / 3400$ & $2 \times 900$ \\
& Keck LRIS & $400 / 8500(7608 \AA)$ & $2 \times 900$
\end{tabular}

\begin{tabular}{llcc}
\hline \multicolumn{4}{c}{ WFI J132813.7-214237 } \\
\hline 2006 Apr 23............ & Keck LRIS & $400 / 3400$ & 3600 \\
& Keck LRIS & $400 / 8500(7608 \AA)$ & 3600 \\
\hline
\end{tabular}

\section{SDSS-SN 15207}

2006 Nov 17........... Gemini S GMOS $\quad 150 / 7170 \quad 2 \times 1750$

NoтE.-In col. (3) we list the grism/grating (number of grooves/blaze angle) and (for the red DBSP and LRIS gratings) the central wavelength. 


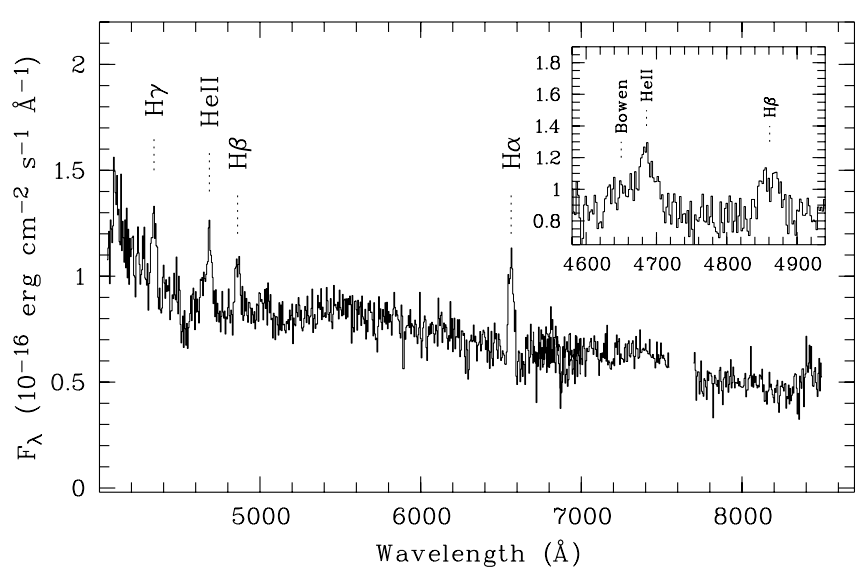

FIG. 3.-DBSP spectrum of ROTSE3 J160213.1-021311.7. The prominent Balmer and He II emission features (labeled) are structured, and a double-peaked shape is visible for $\mathrm{H} \beta$ (inset) and $\mathrm{H} \gamma$. The inset also shows the region around the He II line at $4686 \AA$ revealing a strong Bowen blend of C III, C IV, and N III.

with $\mathrm{H} \alpha, \mathrm{H} \beta$, and $\mathrm{H} \gamma$. The emission lines are clearly broadened with a full width at half-maximum $(45 \pm 2 \AA$ for $\mathrm{H} \alpha)$ suggesting velocities of $>2000 \mathrm{~km} \mathrm{~s}^{-1}$. They are structured and doublepeaked in the cases of $\mathrm{H} \beta$ and $\mathrm{H} \gamma$. In addition, a strong $\mathrm{He}$ II emission line at $4686 \AA$, blended with the Bowen fluorescence (Bowen 1934) of N III, C III, and C IV, is detected. The line properties are summarized in Table 3.

\subsection{Identification}

The decaying light curve matches a fast classical nova (CN) burst. However, the absolute peak magnitude of a fast $\mathrm{CN}$ is $\sim-8 \mathrm{mag}$, which would place the transient at a distance of $1 \mathrm{Mpc}$. The absence of a host galaxy makes this interpretation unlikely.

However, a dwarf nova hypothesis is entirely reasonable and in agreement with the emission spectrum. Dwarf novae (DNe) constitute an important subgroup of cataclysmic variables which undergo outbursts with a brightness increase of 2-8 mag and repetition timescales of days to decades. The large amplitude (>4 mag; see Fig. 2) of ROTSE3 J160213.1-021311.7 along with the outburst duration of $>30$ days resembles a "superoutburst" of SU UMa-like DNe. Superoutburst light curves of these sources typically exhibit a plateau phase with a slow decay of $\sim 1$ mag in 8-10 days after the initial brightening, followed by a steep decline with periodic luminosity modulations, called "superhumps." The observed light curve differs by showing an early flare and a shallower (quasi-exponential) decay at late times. However, we found that this temporal behavior is very similar to the superoutburst of the SU UMa dwarf nova RU Peg in 1981 (la Dous et al. 1985). Although our data are too sparse to detect clear evidence for superhumps, the rebrightenings and variability on short timescales (see Fig. 2, inset) could be interpreted as signatures of such.

TABLE 3

ROTSE3 J160213.1-021311.7 Emission-Line ChARACTERISTICS

\begin{tabular}{|c|c|c|c|}
\hline Species & $\begin{array}{c}\text { Flux } \\
\left(10^{-15} \mathrm{erg} \mathrm{cm}^{-2} \mathrm{~s}^{-1}\right)\end{array}$ & $\begin{array}{l}\text { EW } \\
(\AA)\end{array}$ & $\begin{array}{l}\text { FWHM } \\
(\AA)\end{array}$ \\
\hline $\mathrm{H} \gamma \quad \lambda 4340 \ldots \ldots \ldots \ldots$ & $1.6 \pm 0.3$ & $19 \pm 4$ & $42 \pm 7$ \\
\hline 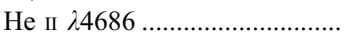 & $1.9 \pm 0.3$ & $25 \pm 5$ & $42 \pm 5$ \\
\hline $\mathrm{H} \beta \quad \lambda 4861 \ldots \ldots \ldots \ldots \ldots \ldots \ldots \ldots \ldots$ & $1.7 \pm 0.2$ & $22 \pm 5$ & $41 \pm 7$ \\
\hline Н $\alpha$ $\lambda 6563$ & $2.6 \pm 0.2$ & $58 \pm 5$ & $45 \pm 2$ \\
\hline
\end{tabular}

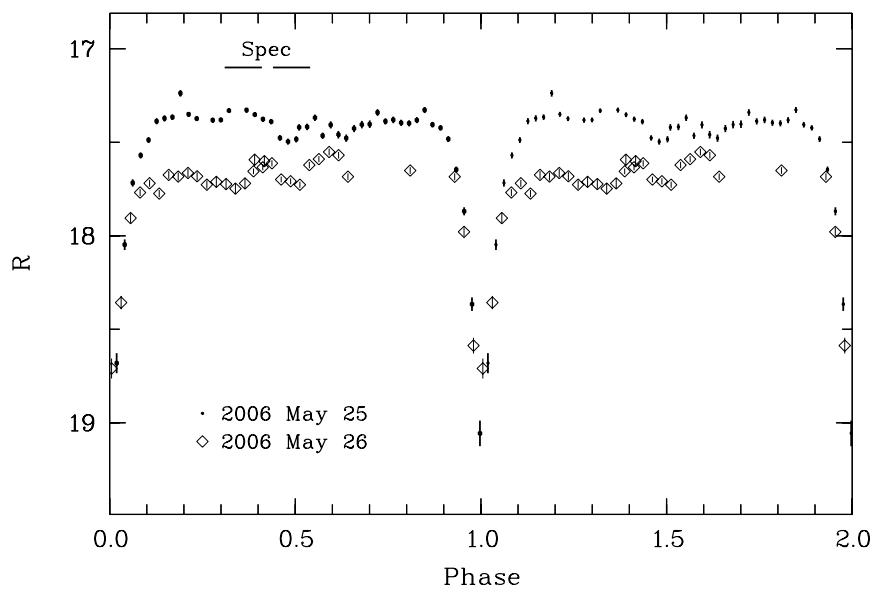

FIG. 4.-Phase-folded P60 light curves of WFI J161953.3+031909 using the ephemeris given in eq. (1) shown twice for clarity. Data taken on May 25 ( filled circles) and 26 (open diamonds) reveal alterations of the out-of-eclipse variability, as well as a changes of the overall brightness level of up to $0.5 \mathrm{mag}$. Additional observations were performed on 2006 May 12, 13, 15, 16, and 17. Horizontal dashes mark the phases of the Keck LRIS spectroscopy observations (see Fig. 5). [See the electronic edition of the Journal for a color version of this figure.]

The spectrum shows strong similarities to IY UMa (an SU UMa DN) during its superoutburst in 2000 (Rolfe et al. 2005). The large flux ratio of $\mathrm{He}$ II $/ \mathrm{H} \beta \sim 1.2$ is also a popular indicator for a highly magnetic $\mathrm{CV}$, e.g., a polar or an intermediate polar. However, the temporal behavior of ROTSE3 J160213.1021311.7 with its large amplitude outburst from a long-lasting quiescent state is entirely inconsistent with the long-term variability expected for a magnetic CV. In addition, the doublepeaked emission lines and lack of pronounced cyclotron lines clearly provide further evidence that this system is a typically disk CV.

Finally, we note the large $g-R$ color $(\sim 1)$ could suggest a cold accretion disk in the system resulting from a low mass accretion rate. A low mass transfer is reminiscent of cataclysmic variables in the period gap between 2 and $3 \mathrm{hr}$ (e.g., Robinson 1983; Ritter \& Kolb 1995), which extents to $\sim 4$ hr for dwarf novae (Shafter et al. 1986). Thus, we conjecture that ROTSE3 J160213.1-021311.7 is a hibernating (Shara et al. 1986) SU UMa dwarf nova.

\section{WFI J161953.3+031909}

\subsection{Discovery}

In 1999, a transient and variability survey (dedicated to the search for orphan afterglows) was performed at the MPG/ESO $2.2 \mathrm{~m}$ telescope equipped with the Wide Field Imager (WFI). Twelve square degrees were monitored in up to 25 nights down to a limiting magnitude of $R=23$ (Rau et al. 2006). The search netted four candidates after filtering for moving or known solar system objects and known variables. For two sources, a classification as a CV and a flare star were suggested based on their temporal behavior. Follow-up observations for remaining two will be described here and in $\S 4$.

WFI J161953.3+031909 is an uncataloged transient found in the WFI survey when it showed a brightening from a persistent $R=19.9$ to 17.5 within 2 days on 1999 June 17.14 UT. Based on the subsequent optical decay and the detection of a faint $\mathrm{X}$-ray source in the ROSAT All-Sky Survey, the source was preliminarily classified as a dwarf nova of the SU UMa class. 


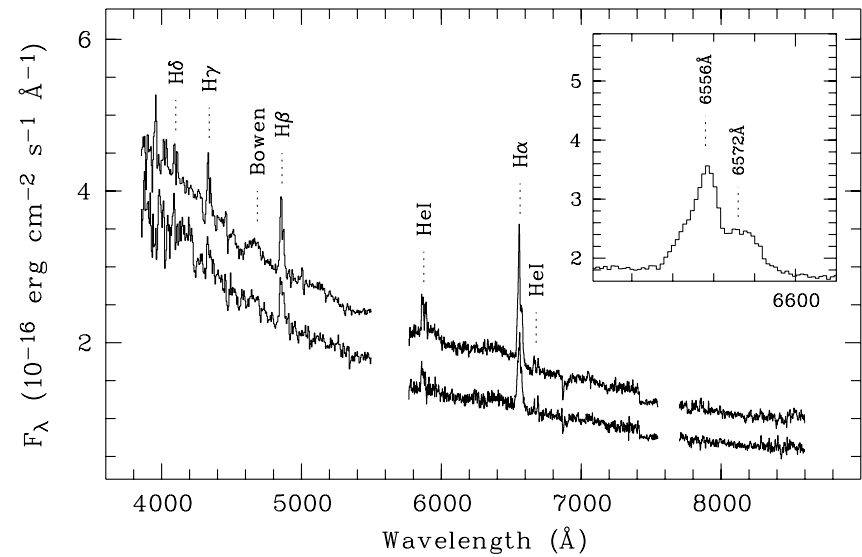

FIG. 5.-LRIS spectra of WFI J161953.3+031909 taken 2006 April 22 at orbital phases $\sim 0.36$ (bottom) and $\sim 0.49$ (top). Prominent Balmer and He I emission lines are indicated. The emission lines show a double-peaked structure, which is shown for $\mathrm{H} \alpha$ (phase $~ 0.49$ ) in the inset. In addition, a prominent Bowen blend is seen at phase $\sim 0.49$ shortward of $\mathrm{H} \beta$. [See the electronic edition of the Journal for a color version of this figure.]

\subsection{Photometry}

Photometry was obtained in seven epochs in 2006 May with the P60. A total of $220 R$-band images with exposures of $150 \mathrm{~s}$ were taken. The photometric calibration was tied to the original WFI discovery image (see Rau et al. 2006 for details). Relative photometry was applied to correct the series of P60 observations to a common reference system. The light curve revealed periodic occultations (Fig. 4), and we derived a firm ephemeris from the timing of six individual eclipses as

$$
\operatorname{HMJD}\left(T_{\text {ecl }}\right)=53880.2503(12)+E 0.099401(9),
$$

with the numbers in parentheses giving the uncertainty in the last digits. The accumulated cycle count between the WFI and P60 observations is too large to derive a unique period solution from 1999 to 2006.

\subsection{Spectroscopy}

Spectroscopy was performed with the Low Resolution Imager and Spectrograph (LRIS; Oke et al. 1995) mounted on the Keck I 10 m telescope (see Table 2). The LRIS light beam is split by a dichroic, and spectra were obtained in the blue and red channels simultaneously. We obtained two 900 s spectra with midexposure times corresponding to orbital phases of $\sim 0.36$ and $\sim 0.49$. The spectra show blue continua with a number of strong emission features which we identify with Balmer emis- sion $(\mathrm{H} \alpha-\mathrm{H} \gamma)$ and $\mathrm{He}$ I at 5876 and $6678 \AA$. All lines are doublepeaked and clearly broadened with respect to the instrumental line width (see Fig. 5, inset). In addition, a broad emission feature centered at $\lambda=4665 \AA$ is found at phase 0.49 . We associate this with a Bowen blend of N III, C III, and C IV. A summary of the line properties is given in Table 4.

\subsection{Identification}

The periodic light curve and the spectrum resemble those of a dwarf nova in quiescence. The detection of deep orbital eclipses (Fig. 4) together with the double-peaked emission lines indicate a high inclination of the system. Out of eclipse, the light curve appears flatter than typically observed in eclipsing dwarf novae. In addition, no strong asymmetry is seen between ingress and egress, suggesting that the hot spot, the region where the gas stream collides with the accretion disk, would be weaker than usual. This is in agreement with the low mass transfer rate assumed for CVs in the period gap, in which WFI J161953.3+ 031909 falls.

The double-peaked emission lines (Fig. 5), the absence of pronounced cyclotron features, and the nondetection of He II indicate a disk system. The detection of the Bowen blend around $4665 \AA$ a bears evidence of irradiation in the disk or the secondary. However, He II emission, which leads to the Bowen fluorescence, is not detected. This suggests a considerable He II opacity.

The orbital period of $\sim 143$ minutes implies that observations taken at the same UT but over different nights will result in the source being observed at nearly identical orbital phases. In retrospect, we now realize that the original survey data were obtained at phases at which the object exhibited extreme brightness. The maximum brightness in the WFI detections of $R=17.5$ corresponds to the median brightness out of eclipse (see Fig. 4), and the (falsely interpreted) quiescent brightness of $R=19.9$ is similar to what is observed during the eclipse.

To summarize, we found no evidence for a dwarf nova outburst of WFI J161953.3+031909 and thus are left with the rough classification of the source as a disk CV. Indeed, the brighter quiescent magnitude than previously thought implies $L_{\mathrm{X}} / L_{\mathrm{opt}} \sim$ 0.1 , consistent with a larger variety of CVs.

\section{WFI J132813.7-214237}

\subsection{Discovery}

The source was detected on 1999 June 26.07 UT, at a maximum brightness of $R=19.9$. As a result of the scheduling, no observations could be obtained during a subsequent decay. However, a faint uncataloged and persistent object $(R=21.3)$ was found, offset by $0.8^{\prime \prime}$ from the location of the transient source.

TABLE 4

WFI J161953.3+031909 EMISSION-Line CharaCteristics

\begin{tabular}{|c|c|c|c|c|}
\hline \multirow[b]{2}{*}{ SPECIES } & \multicolumn{2}{|c|}{ Phase $\sim 0.36$} & \multicolumn{2}{|c|}{ PHASE $\sim 0.49$} \\
\hline & $\begin{array}{c}\text { Flux } \\
\left(10^{-15} \mathrm{erg} \mathrm{cm}^{-2} \mathrm{~s}^{-1}\right)\end{array}$ & $\begin{array}{l}\text { EW } \\
(\AA)\end{array}$ & $\begin{array}{c}\text { Flux } \\
\left(10^{-15} \mathrm{erg} \mathrm{cm}^{-2} \mathrm{~s}^{-1}\right)\end{array}$ & $\begin{array}{l}\text { EW } \\
(\AA)\end{array}$ \\
\hline 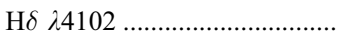 & $<1.2$ & $<5$ & $1.4 \pm 0.2$ & $-3 \pm 1$ \\
\hline 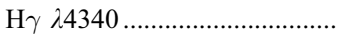 & $1.5 \pm 0.5$ & $6 \pm 2$ & $2.7 \pm 0.2$ & $4 \pm 1$ \\
\hline 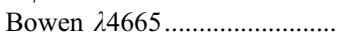 & $<2.2$ & $<9$ & $5.5 \pm 0.5$ & $15 \pm 5$ \\
\hline 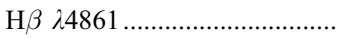 & $2.8 \pm 0.3$ & $13 \pm 1$ & $3.7 \pm 0.2$ & $13 \pm 1$ \\
\hline 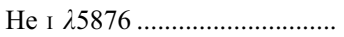 & $1.0 \pm 0.2$ & $7 \pm 1$ & $1.5 \pm 0.4$ & $6 \pm 1$ \\
\hline 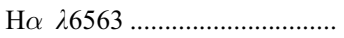 & $3.9 \pm 0.3$ & $34 \pm 3$ & $5.4 \pm 0.3$ & $34 \pm 3$ \\
\hline 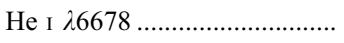 & $0.4 \pm 0.1$ & $3.4 \pm 0.4$ & $0.6 \pm 0.1$ & $3.6 \pm 0.5$ \\
\hline
\end{tabular}




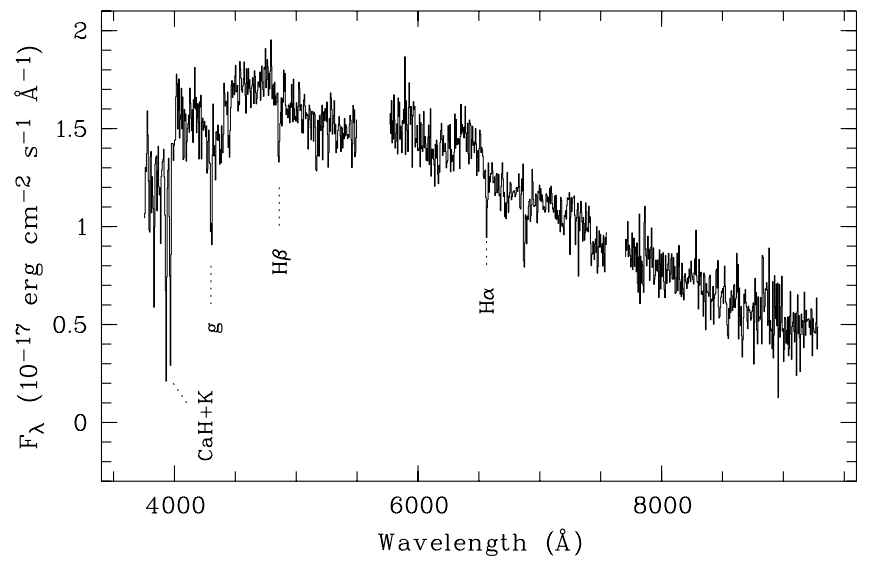

FIG. 6.-LRIS spectrum of the quiescent counterpart to WFI J132813.7214237 taken 2006 April 23. Prominent absorption features are indicated.

This apparent association allowed for a possible extragalactic origin of the transient with the persistent source being the underlying host galaxy. It was therefore interpreted as the best candidate for an orphan afterglow found in the survey. However, given the sparse data coverage, other interpretations (extragalactic, Galactic, and even solar system) could not be excluded.

\subsection{Spectroscopy}

Spectroscopy of the of the persistent source associated with WFI J132813.7-214237 was performed with LRIS. We find strong absorption features which we identify with $\mathrm{Ca} \mathrm{H}$ and $\mathrm{K}$, $g$ band, $\mathrm{H} \beta$, and $\mathrm{H} \alpha$ (Fig. 6). The narrow lines allow us to estimate the velocity with respect to the local standard rest frame to within $0 \pm 50 \mathrm{~km} \mathrm{~s}^{-1}$. No significant emission lines are detected.

\subsection{Identification}

The spectrum is consistent with that of a Galactic $G$ star. Thus, we can rule out an extragalactic origin for the persistent source. As a result, there is little justification for assuming that the transient source was extragalactic. Instead the detection was likely the misleading alliance of a moving solar system object superimposed on a background star. The position in the ecliptic plane (ecliptic coordinates $\lambda=208.5^{\circ}, \beta=-11.6^{\circ}$ ) supports this scenario.

\section{SDSS-SN 15207}

\subsection{Discovery}

SDSS-SN 15207 was discovered on 2006 October 11 as part of the Sloan Digital Sky Survey (SDSS) search for supernovae, at $\alpha_{\mathrm{J} 2000.0}=20^{\mathrm{h}} 58^{\mathrm{m}} 43.45^{\mathrm{s}}, \delta_{\mathrm{J} 2000.0}=01^{\circ} 02^{\prime} 47.0^{\prime \prime}$. SDSS imaging prior to the eruption shows no underlying host galaxy to a limit of $r=24$. The SDSS optical light curve ${ }^{8}$ showed a peak magnitude of $r=19.8$ on October 12 followed by a decline by 1.2 mag over 5 days. Continued monitoring with P60 during the first 80 days did not detect additional outbursts to a limiting magnitude of $r=22$.

\subsection{Spectroscopy}

We obtained a spectrum with the Gemini Multi Object Spectrograph (GMOS) on Gemini South on 2006 November 17 (Table 2). At this time, the event had faded to $r \sim 23$. This resulting low signal-to-noise ratio spectrum (Fig. 7) shows a blue con-

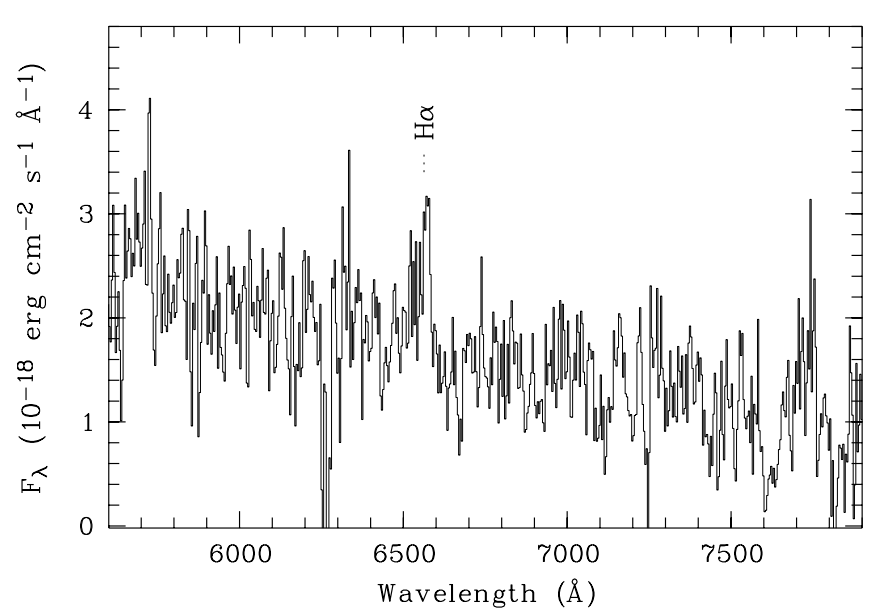

FIG. 7.-Gemini GMOS spectrum of SDSS-SN 15207 obtained 2006 November 17,36 days after the discovery.

tinuum and a single emission line $\left(\mathrm{FWHM}=1000 \pm 200 \mathrm{~km} \mathrm{~s}^{-1}\right)$, which we identify with $\mathrm{H} \alpha$. The line centroid suggest a Galactic origin.

We also obtained a $4.77 \mathrm{ks}$ exposure with the X-ray telescope aboard Swift on 2006 November 4. No counterpart was found with a $2 \sigma$ flux limit of $>6 \times 10^{-14} \mathrm{ergs} \mathrm{cm}^{-2} \mathrm{~s}^{-1}(0.2-10 \mathrm{keV})$.

\subsection{Identification}

The peak magnitude of this transient rules out the possibility that it is a nova in the Milky Way. The optical light curve, in particular, the steep decline of $1.2 \mathrm{mag}$ in 5 days and $1.6 \mathrm{mag}$ in 9 days, does not suggest a typical supernova, either. On the other hand, the observations are consistent with a superoutburst ( $>4 \mathrm{mag}$ ) of a Galactic dwarf nova. The nondetection of the quiescent counterpart to $r=24$ and the lack of significant foreground extinction in the optical colors and spectrum suggest that SDSS-SN 15207 has to be located at a relatively large distance from the earth. Assuming an absolute quiescence magnitude of $R=12$ (white dwarf with $T=11,000 \mathrm{~K}, M=0.6 M_{\odot}$; Bergeron et al. 1995), the lower limit on the distance is $2.5 \mathrm{kpc}$.

\section{DISCUSSION AND CONCLUSION}

Here we reported on photometric and spectroscopic follow-up observations for four recently communicated transient sources. We found that WFI J132813.7-214237, the best orphan afterglow candidate detected in the WFI survey (Rau et al. 2006), was with high probability a misidentified solar system object spatially coincident with a background source. Next, the potential outburst light curve of a second source from the same survey, WFI J161953.3+031909, was recognized as serendipitous observations in and out of eclipse of a high-inclination cataclysmic variable. Finally, ROTSE3 J160213.1-021311.7, discovered by ROTSE-III (Rykoff et al. 2006), and (potentially) SDSSSN 15207 were identified as the superoutbursts of SU UMa-like dwarf novae.

In the following, we restrain from exploring the two WFI sources any further, except noting again the interesting coincidences which had previously lured us to incorrect conclusions. Instead, we want to address the implications of the faint quiescent magnitudes of ROTSE3 J160213.1-021311.7 $(g \sim 23)$ and SDSS-SN $15207(r>24)$. We start by noting that a very similar event $\left(1955+22 \mathrm{C}\right.$ VAR VUL $\left.05^{9}\right)$ was discovered in 
TABLE 5

Dwarf Novae Number Counts and Outburst Rates

\begin{tabular}{|c|c|c|c|c|}
\hline \multirow[b]{2}{*}{ Galactic Latitude } & \multicolumn{2}{|c|}{$N_{\mathrm{DN}}^{\mathrm{a}}$} & \multicolumn{2}{|c|}{$N_{\mathrm{SO}}{ }^{\mathrm{b}}$} \\
\hline & $\rho=3 \times 10^{-5} \mathrm{pc}^{-3}$ & $\rho=10^{-3} \mathrm{pc}^{-3}$ & $\rho=3 \times 10^{-5} \mathrm{pc}^{-3}$ & $\rho=10^{-3} \mathrm{pc}^{-3}$ \\
\hline$|b|<10^{\circ} \ldots \ldots \ldots \ldots$ & $\sim 1.7 \times 10^{3}$ & $\sim 5 \times 10^{4}$ & $\sim 40$ & $\sim 1.5 \times 10^{3}$ \\
\hline $10^{\circ}<|b|<45^{\circ}$ & $\sim 1.8 \times 10^{3}$ & $\sim 5 \times 10^{4}$ & $\sim 40$ & $\sim 1.5 \times 10^{3}$ \\
\hline$|b|>45^{\circ}$ & $\sim 5 \times 10^{2}$ & $\sim 1.5 \times 10^{4}$ & $\sim 15$ & $\sim 4 \times 10^{2}$ \\
\hline
\end{tabular}

Notes.- Note that the theoretical DN density includes a large population of events with superoutburst cycles of decades. Thus, the presented numbers are optimistic. Results will change if a different scale height (e.g., 150 pc; Patterson 1984) is adopted.

a Total number of dwarf novae within the considered volume. See text for details.

${ }^{\mathrm{b}}$ Number of superoutbursts in an all-sky snapshot.

2005 August with 15.8 mag at maximum and an amplitude of at least 9 mag.

This quiescent faintness of these three events indicates an important factor for future wide-field transient searches, namely, dwarf novae which stay most of the time below the limiting magnitude of a survey but appear as new transient sources during outbursts. This includes CVs which appear faint in quiescence because of large distance or Galactic extinction and intrinsically low-magnitude systems. The latter group is dominated by systems with low mass transfer, typical for CVs in the period gap and in hibernation (Shara et al. 1986). As a matter of fact, the vast majority of nonmagnetic CVs with $P_{\text {orb }}<3 \mathrm{hr}$ are dwarf novae ( $\sim 83 \%)$, while this is true only for $\sim 36 \%$ with $P_{\text {orb }}>3 \mathrm{hr}$ (Aungwerojwit et al. 2006).

As an example, let us consider the proposed $3 \pi$ sr survey with Pan-STARRS 1 (PS1), which is expected to reach a limiting magnitude of $R \sim 23.2$ and $=24.9$ in a single $30 \mathrm{~s}$ image and combined images over $3 \mathrm{yr}$, respectively. In such a survey, sources similar to ROTSE3 J160213.1-021311.7 at twice the distance would no longer be detectable in quiescence, but only during the rare outburst periods. This directly leads to the question of how important these events will be in future wide-field surveys.

The expected number of DNe events in an all-sky snapshot can be obtained rather crudely, given the large number of involved parameters (e.g., intrinsic quiescent magnitude, superoutburst amplitude, and superoutburst cycle). Let the quiescent absolute magnitude of the DN be $M_{R} \sim 12$ (Bergeron et al. 1995). For the volume distribution we assume a local scale height of $300 \mathrm{pc}$ perpendicular to the Galactic disk and isotropy in the radial direction in the disk. Furthermore, we consider for simplicity an upper limit for the distance in the Galactic plane of $2 \mathrm{kpc}$, above which extinction will dominate at low Galactic latitude. For the observed local density ${ }^{10}$ of dwarf novae we use $\rho \sim 3 \times 10^{-5} \mathrm{pc}^{-3}$ (Schwope et al. 2002), and theory allows for even higher values $\left(10^{-4}\right.$ to $10^{-3} \mathrm{pc}^{-3}$; Kolb 1993 ; de Kool \& Ritter 1993). The resulting number of expected dwarf novae in the considered volume, independent of their activity, is given in Table 5 for three different ranges of Galactic latitude.

How many of those will be in superoutburst at any given time? Assuming a mean cycle duration of $1 \mathrm{yr}$ and a mean plateau length of 10 days we find that between 40 and $1.5 \times 10^{3}$ events, depending on the Galactic latitude and assumed space density, can be expected in an all-sky snapshot with maximum depth. Most of these $(\sim 86 \%)$ will be at low and medium Galactic lat-

\footnotetext{
${ }^{10}$ Note that the observationally derived space densities are uncertain by at least a factor of 10 in both directions. A detailed assessment of possible selection effects in surveys can be found in Patterson (1984).
}

itude, and only a small fraction $(\sim 14 \%)$ are expected to occur at $|b|>45^{\circ}$.

The space density of CVs decreases rapidly with distance from the Galactic plane. Similarly, at low Galactic latitude extinction limits the observable volume. As a result, the number of dwarf novae with quiescent brightness below the survey limiting magnitude (here $R \sim 25$ ) will be negligible. However, shallower surveys will detect less quiescent systems as indicated in Figure 8. Thus, these surveys will mainly be contaminated by dwarf novae superoutbursts with undetected quiescent counterparts.

The yearly all-sky rate of less than $4 \times 10^{4}$ detectable superoutbursts is in the range of the local volume rates $\left(\mathrm{Gpc}^{-3} \mathrm{yr}^{-1}\right)$ for core-collapse SNe $\left(\sim 5 \times 10^{4}\right.$; Cappellaro et al.1999) but considerably lower than the rates of novae $\left(\sim 10^{8}\right.$; Kulkarni \& Rau 2006) and Galactic M-dwarf flares $\left(10^{8} \mathrm{yr}^{-1}\right.$; Kulkarni $\&$ Rau 2006). In addition, one has to remember that the theoretical CV space densities typically imply a large fraction of systems with cycle periods of years to decades (e.g., WZ Sge). Thus, the yearly all-sky rate is probably much less than value given above. This suggests that dwarf nova superoutbursts will not produce a dominant contamination in future wide-field surveys. However, they will still be of concern for observers mining the optical transient sky.

How can the small number of dwarf novae outbursts be distinguished in practice from other kinds of interesting transients?

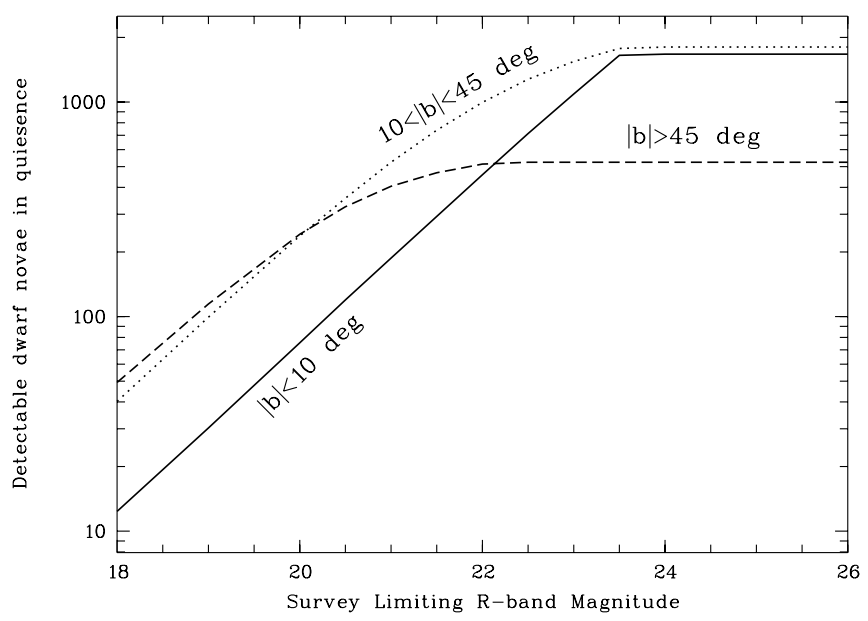

FIG. 8.-Predictions for the number of dwarf novae which can be detected in a survey with a given limiting $R$-band magnitude. The solid, dashed, and dotted lines show the expectations for three ranges of Galactic latitude. Note that the sharp turnover for $|b|<10^{\circ}$ is caused by the distance limit in the Galactic plane ( $2 \mathrm{kpc}$ ), which was assumed to account for foreground extinction. At $|b|>45^{\circ}$ nearly all quiescent systems will be detected above $R=22$. 
The most important factors will be the choice of cadence and the field selection. A good sign for a DN will be a location close to the Galactic disk or inner Galaxy. Indeed, the positions of ROTSE3 J160213.1-021311.7 $\left(l=8^{\circ}, b=35^{\circ}\right)$ and of $1955+$ 22C VAR VUL $05\left(l=60^{\circ}, b=-4^{\circ}\right)$ are consistent with being part of the disk population. However, the Galactic plane is known to harbor plentiful of variable and transient sources. Choosing the right observing strategy (cadence and filter) will help to distinguish between short-lived phenomena (e.g., flare stars) and dwarf novae. The main confusion at high Galactic latitude will be by novae and supernovae. Novae, however, will be distinguishable by their enormous brightness (if Galactic) or by their association with a nearby galaxy. Supernovae may be similarly identified by their host galaxies. Spectroscopy of the remaining candidates would require significant amounts of time on large telescopes and is thus not a feasible approach. Photometric colors vary considerably from one event to another and will therefore not be usable as reliable indicators, either. The best confirmation, however, can be obtained by photometric follow-up searches for the expected superhump structures.

We close this paper noting the role of future large surveys for our general understanding of cataclysmic variables. Complete wide-area surveys on long timescales will allow the in-depth study of the distribution of orbital periods and outburst behaviors (amplitudes and cycles). This will provide important information on the evolution of CVs and the processes of mass accretion in these systems.

We acknowledge helpful discussions with J. Greiner, E. Mason, and L. Bildsten. We thank M. Sako and A. Becker for the timely alert about the eruption of SDSS-SN 15207. Furthermore, we thank the anonymous referee for constructive comments. This work is supported in part by grants from the National Science Foundation and NASA.
Astier, P., Guy, J., \& Regnault, N. 2006, A\&A, 447, 31

Aungwerojwit, A., et al. 2006, A\&A, 455, 659

Becker, A. C., et al. 2004, ApJ, 611, 418

Bergeron, P., Wesemael, F., \& Beauchamp, A. 1995, PASP, 107, 1047

Bowen, I. S. 1934, PASP, 46, 146

Cappellaro, E., Evans, R., \& Turatto, M. 1999, A\&A, 351, 459

de Kool, M., \& Ritter, H. 1993, A\&A, 267, 397

Kaiser, N., et al. 2002, Proc. SPIE, 4836, 154

Kolb, U. 1993, A\&A, 271, 149

Kulkarni, S. R., \& Rau, A. 2006, ApJ, 644, L63

la Dous, C., Verbunt, F., \& Schoembs, R. 1985, MNRAS, 212, 231

Landolt, A. U. 1992, AJ, 104, 340

Malacrino, F., \& Atteia, J.-L. 2006, GCN Circ. 4705, http:/gcn.gsfc.nasa.gov/ $\mathrm{gcn} / \mathrm{gcn} 3 / 4705 . \mathrm{gcn} 3$

Malacrino, F., et al. 2006, A\&A, 459, 465

Oke, J. B., et al. 1995, PASP, 107, 375

Patterson, J. 1984, ApJS, 54, 443

Rau, A., Greiner, J., \& Schwarz, R. 2006, A\&A, 449, 79

\section{REFERENCES}

Ritter, H., \& Kolb, U. 1995, in X-Ray Binaries, ed. W. H. G. Lewin, J. van Paradijs, \& E. P. J. van den Heuvel (Cambridge: Cambridge University Press), 578

Robinson, E. L. 1983, in Cataclysmic Variables and Related Objects, ed. M. Livio \& G. Shaviv (Dordrecht: Reidel), 1

Rolfe, D. J., Haswell, C. A., \& Abbott, T. M. C. 2005, MNRAS, 357, 69

Rykoff, E. S., Aretakis, J., \& Guver, T. 2006, ATel, 824

Rykoff, E. S, et al. 2005, ApJ, 631, 1032

Sako, M., et al. 2005, preprint (astro-ph/0504455)

Schlegel, D. J., Finkbeiner, D. P., \& Davis, M. 1998, ApJ, 500, 525

Schmidt, B. P., Keller, S. C., Francis, P. J., \& Bessell, M. S. 2005, BAAS, 37, 457

Schwope, A. D., Brunner, H., Buckley, D., Greiner, J., Heyden, K. v. d., Neizvestny, S., Potter, S., \& Schwarz, R. 2002, A\&A, 396, 895

Shafter, A. W., Wheeler, J. C., \& Cannizzo, J. K. 1986, ApJ, 305, 261

Shara, M. M., Livio, M., Moffatt, A. F. J., \& Orio, M. 1986, ApJ, 311, 163

Tyson, A. 2005, in ASP Conf. Ser. 339, Observing Dark Energy, ed. S. C. Wolff \& T. R. Lauer (San Francisco: ASP), 95 